\title{
Cryptic breakpoint identified by whole- genome mate-pair sequencing in a rare paternally inherited complex chromosomal rearrangement
}

Constantia Aristidou ${ }^{1,2}$, Athina Theodosiou' ${ }^{1}$, Andria Ketoni ${ }^{1}$, Mads Bak ${ }^{3}$, Mana M. Mehrjouy ${ }^{3}$, Niels Tommerup ${ }^{3}$ and Carolina Sismani ${ }^{1,2^{*}}$ (D)

\begin{abstract}
Background: Precise characterization of apparently balanced complex chromosomal rearrangements in nonaffected individuals is crucial as they may result in reproductive failure, recurrent miscarriages or affected offspring.

Case presentation: We present a family, where the non-affected father and daughter were found, using FISH and karyotyping, to be carriers of a three-way complex chromosomal rearrangement $[t(6 ; 7 ; 10)(q 16.2 ; q 34 ; q 26.1)$, de novo in the father]. The family suffered from two stillbirths, one miscarriage, and has a son with severe intellectual disability. In the present study, the family was revisited using whole-genome mate-pair sequencing. Interestingly, whole-genome mate-pair sequencing revealed a cryptic breakpoint on derivative (der) chromosome 6 rendering the rearrangement even more complex. FISH using a chromosome (chr) 6 custom-designed probe and a chr10 control probe confirmed that the interstitial chr6 segment, created by the two chr6 breakpoints, was translocated onto der(10). Breakpoints were successfully validated with Sanger sequencing, and small imbalances as well as microhomology were identified. Finally, the complex chromosomal rearrangement breakpoints disrupted the SIM1, GRIK2, CNTNAP2, and PTPRE genes without causing any phenotype development.
\end{abstract}

Conclusions: In contrast to the majority of maternally transmitted complex chromosomal rearrangement cases, our study investigated a rare case where a complex chromosomal rearrangement, which most probably resulted from a Type IV hexavalent during the pachytene stage of meiosis I, was stably transmitted from a fertile father to his nonaffected daughter. Whole-genome mate-pair sequencing proved highly successful in identifying cryptic complexity, which consequently provided further insight into the meiotic segregation of chromosomes and the increased reproductive risk in individuals carrying the specific complex chromosomal rearrangement. We propose that such complex rearrangements should be characterized in detail using a combination of conventional cytogenetic and NGS-based approaches to aid in better prenatal preimplantation genetic diagnosis and counseling in couples with reproductive problems.

Keywords: CCR, Familial, Paternal transmission, WG-MPS, Cryptic breakpoint, Reproductive problems

\footnotetext{
* Correspondence: csismani@cing.ac.cy

'Department of Cytogenetics and Genomics, The Cyprus Institute of

Neurology and Genetics, Nicosia, Cyprus

${ }^{2}$ The Cyprus School of Molecular Medicine, The Cyprus Institute of Neurology

and Genetics, Nicosia, Cyprus

Full list of author information is available at the end of the article
}

(c) The Author(s). 2018 Open Access This article is distributed under the terms of the Creative Commons Attribution 4.0 International License (http://creativecommons.org/licenses/by/4.0/), which permits unrestricted use, distribution, and reproduction in any medium, provided you give appropriate credit to the original author(s) and the source, provide a link to the Creative Commons license, and indicate if changes were made. The Creative Commons Public Domain Dedication waiver (http://creativecommons.org/publicdomain/zero/1.0/) applies to the data made available in this article, unless otherwise stated. 


\section{Background}

Complex chromosomal rearrangements (CCRs) are generally defined as structural rearrangements that involve more than two chromosome breaks resulting in exchanges of chromosomal segments [1]. The occurrence of constitutional CCRs is rare with approximately 250 cases reported so far $[2,3]$. The majority of apparently balanced CCR carriers are phenotypically normal [2]. However, affected CCR carriers have been previously reported presenting with intellectual disability or other clinical phenotypes. These develop mainly through dosage-sensitive gene disruption [4], disruption of cis-regulatory elements, thus, affecting the expression of disease-candidate genes via long-range position effect $[5,6]$, presence of cryptic imbalances near the breakpoints or elsewhere in the genome [7-9], as well as unmasking of recessive variants by the CCR on the intact chromosomes $[1,10]$. In addition, male infertility [11], recurrent miscarriages [12], as well as stillbirths are common reproductive problems associated with otherwise healthy couples carrying apparently balanced CCRs.

Pregnancy outcomes in CCR carriers have been investigated first by Gorski et al. [13]; the risk for miscarriages and abnormal pregnancies in couples with CCRs was estimated to be at 48.3 and $53.7 \%$, respectively [13]. However, these are general guidelines and since most CCRs are unique in each carrier or family, it is strongly recommended that individual CCRs should be investigated separately [2]. Accurate prediction of the phenotypic outcome of each pregnancy and reproductive risk estimation is challenging in the case of CCRs because of the different malsegregation patterns and recombination events that can occur resulting in unbalanced gametes $[2,3]$. In addition, the higher the complexity of a CCR (i.e. increasing number of chromosomes and breakpoints involved in a rearrangement) and the possibility of recombination events, the higher the percentage of unbalanced gamete generation and the risk for having an affected offspring [2, 3]. Therefore, precise characterization of balanced CCRs is crucial in terms of estimating a more accurate percentage for reproductive risk and abnormal pregnancies, and thus, providing better genetic counseling in couples carrying such complex rearrangements.

High resolution next generation sequencing approaches have been proven fruitful for detailed investigation of CCRs [4]. We have previously demonstrated that whole-genome mate-pair sequencing (WG-MPS) is highly efficient in accurately mapping familial apparently balanced reciprocal translocation breakpoints [14]. Moreover, our group and others have also shown that WG-MPS, often in combination with conventional methods, is a powerful tool for revealing additional complexity in CCR carriers, including chromothripsis rearrangements, that could remain undetected by using only conventional methods with lower resolution (manuscript in preparation) $[15,16]$.
In this study, WG-MPS was applied in order to further characterize and delineate the breakpoints of a de novo CCR involving chromosomes 6,7 , and 10 in a phenotypically normal male with reproductive failure in his family. By revealing the full complexity of the CCR, we aim to provide more precise abnormal pregnancy risk estimations and better genetic counseling in individuals carrying the specific CCR.

\section{Case presentation}

\section{Case report and preliminary analyses}

A family was referred to the Department of Cytogenetics and Genomics, as they suffered from two still births (II:1 and II:4) and one miscarriage (II:3). They also have a son with severe intellectual disability (II:2) and a non-affected daughter (II:5) (Fig. 1a).

Initial chromosomal analysis performed elsewhere using conventional G-banding at the 550-band level detected a de novo chromosomally apparently balanced translocation (ABT) involving chromosomes (chr) 6 and 7 in the non-affected father (I:1) [46,XY,t(6;7)(q16;q34)], while a normal karyotype $[46, \mathrm{XX}]$ was detected in the non-affected mother (I:2). Subsequent Fluorescence In-Situ Hybridization (FISH) analyses by Patsalis et al. [17] revealed cryptic complexity and the involvement of chr10 as well in the rearrangement. At that time, the karyotype of the father was revised as 46,XY,t(6;7;10)(q16.2;q34;q26.1)dn. The non-affected daughter inherited the same CCR [46,XX,t(6;7;10)(q16.2;q34;q26.1)pat], whereas the affected son inherited only der(10) and normal chromosomes 6 and 7 from the father [46,XY,der(10)t(6;7;10)(q16;q34;q26)pat], resulting in a partial 10qter monosomy $(\sim 6 \mathrm{Mb})$ and 7qter trisomy $(\sim 11.5 \mathrm{Mb})$ [17].

\section{Whole-genome mate-pair sequencing}

WG-MPS library preparation, using $1 \mu \mathrm{g}$ DNA from the father and the Nextera Mate-Pair Sample Preparation kit Illumina, San Diego, CA, USA), sequencing on HiSeq2500, WG-MPS data analysis using Burrows-Wheeler Aligner-MEM [18], SVDetect [19], and Integrative Genomics Viewer [20], as well as translocation breakpoint validation with Polymerase Chain Reaction (PCR) and Sanger sequencing were done as previously described [14].

\section{Fluorescence In-Situ Hybridization}

FISH analyses were performed, using a custom-designed FISH probe on $6 \mathrm{q} 16.3$ and a control probe on 10q11.2 (BlueGnome Ltd., Cambridge, United Kingdom), on fixed chromosome suspensions from the father and daughter according to the manufacturer's protocols. 

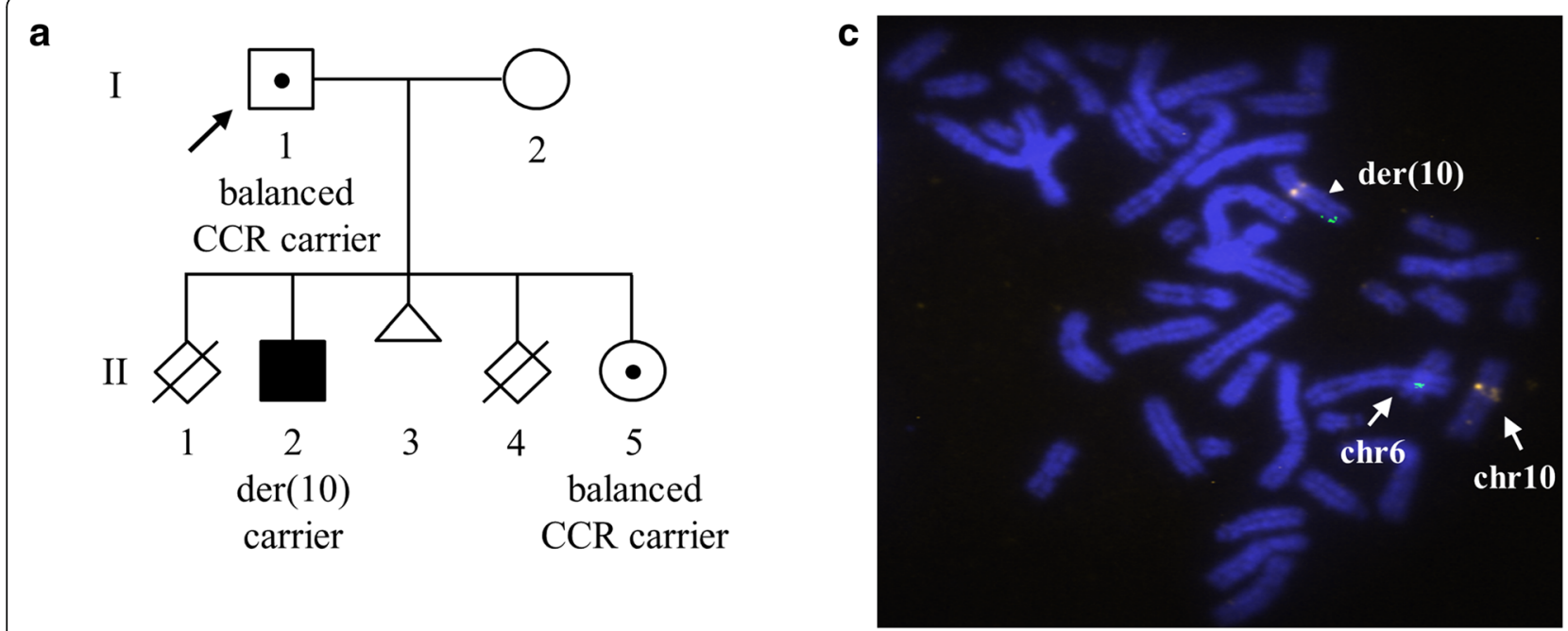

b

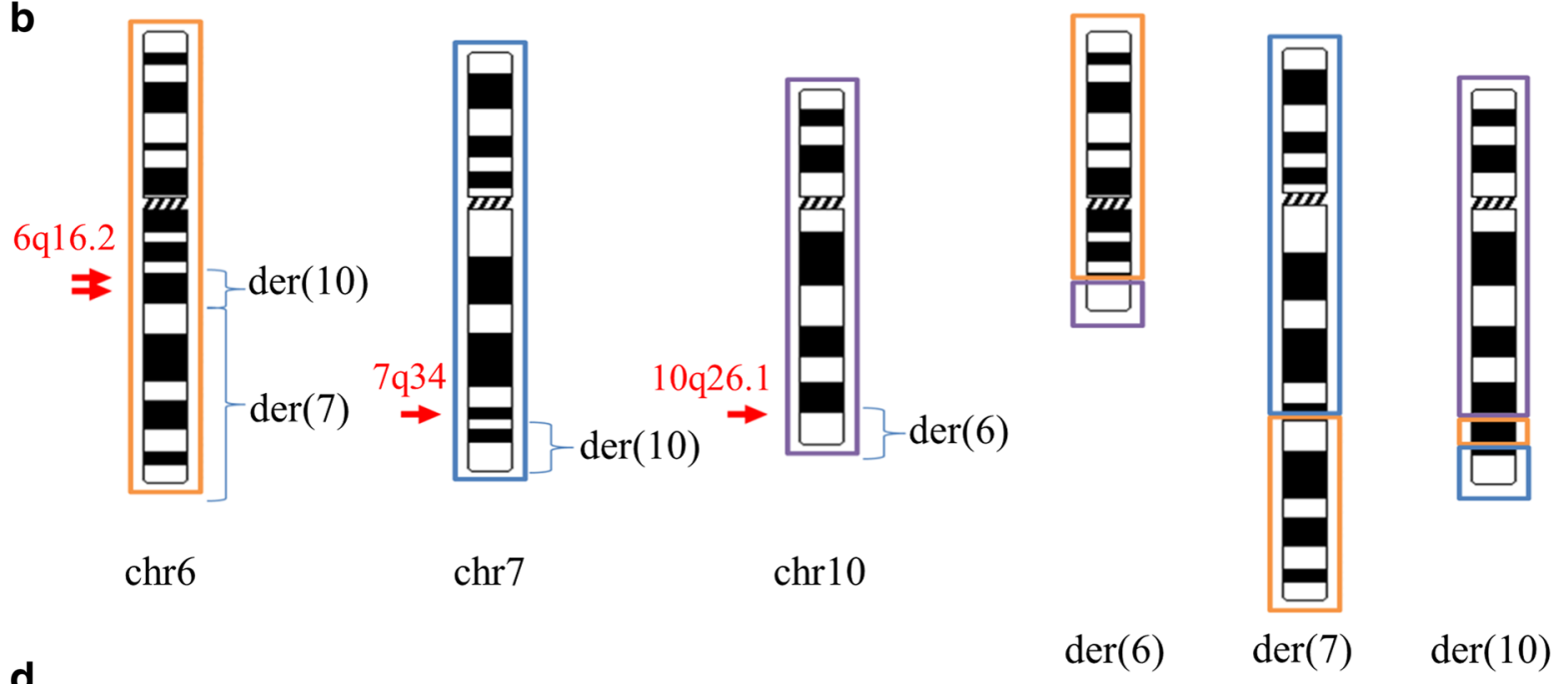

6q16 (+) GACGGAGAGTCTAGACTAGAGAAACCAGTtggtttcatgtagagaaggagttgagag der (6) GACGGAGAGTCTAGACTAGAGAAACCAGTCCTGTCAGCCAAGGCCACTCCCTACAGGA $10 \mathrm{q} 26(+)$ gcatctcactaactcccattcctccctcTCCTGTCAGCCAAGGCCACTCCCTACAGGA 7q34 (+) GGTCTGACTTGTTTAACTTAAAGTCCTTAgactatgtatgtcacaacattcataaaa $\operatorname{der}(7)$ $6 q 16(+)$ GGTCTGACTTGTTAAATTAAAGTCCTTATATTCTCATCTTCCCAAATCCCAGTAGAT taatattacttttcagggagaaagaagtTATTCTCATCTTCCCAAATCCCAGTAGAT

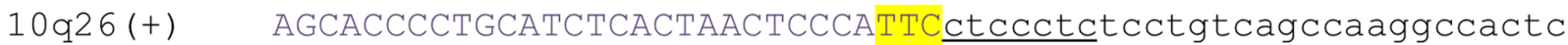
$\operatorname{der}(10) A^{\prime}$ AgCACCCCTGCATCTCACTAACTCCCATTCATGTAGAGAAAGGAGTTGAGAGGCTGGT $6 q 16(+)$ gagtctagactagagaaaccagttggtTTCATGTAGAGAAAGGAGTTGAGAGGCTGGT

6q16(+) ATTACTTTTCAGGGAGAAAGAAGTTATTCTcatcttcccaatcccagtagatttct

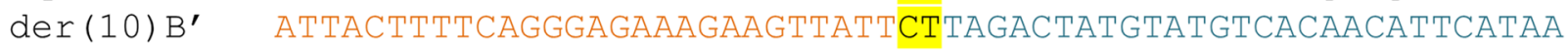
$7 q 34(+) \quad$ ctcggtctgacttgtttaacttaagtcCTTAGACTATGTATGTCACAACATTCATAA

Fig. 1 (See legend on next page.) 
(See figure on previous page.)

Fig. 1 Family Pedigree, Whole-Genome Mate-Pair Sequencing and FISH Results. a Family pedigree depicting the non-affected father (I:1), non-affected daughter (II:5), and affected son (II:2) with severe intellectual disability. The family also suffered from two stillbirths (II:1 and II:4) and one miscarriage (II:3). b ldeograms displaying the normal and derivative chromosomes 6 (orange), 7 (blue) and 10 (purple) (not to scale). The approximate breakpoint positions on 6q16.2, 7q34, and 10q26.1 are indicated by arrows, and the derivative chromosomes onto which each segment is translocated are also shown. c FISH results using a custom-designed probe within 6 q16.3 (green signal) and a control probe within 10q11.22 (orange signal) on metaphase spreads from the non-affected daughter. Both signals are visible on der(10) (arrowhead), and as expected, a green and an orange signal were seen on normal chromosomes 6 and 10 (arrows), respectively. The same results were also observed in the non-affected father (not shown). d CCR breakpoint sequences as identified by WG-MPS and verified by Sanger sequencing. Derivative chromosome sequences (middle line) and matching reference sequences are in capital letters. Microhomology is highlighted, deleted sequences around the breakpoints are underlined, and duplicated sequences are in bold letters

\section{Results}

In the current study, WG-MPS in the father revealed a cryptic translocation breakpoint on chr6, thus rendering the rearrangement even more complex as compared with the three-way CCR identified from the initial karyotype and FISH analyses. In total, four translocation junctions were identified by WG-MPS; two on chr6 ( 1.37 Mb apart from each other), one on chr7 and one on chr10 (Table 1). The interstitial segment created from the additional cryptic translocation breakpoint on chr6 was translocated on der(10) proximal to the 7q34-qter segment (Fig. 1b); this was validated with FISH using a custom designed probe within the chr6 interstitial segment and a control probe on chr10 (Fig. 1c).

After reconstructing all derivative chromosomes (Fig. 1b), breakpoints were successfully mapped to the base-pair level by Sanger sequencing in both non-affected father and daughter using the same PCR primer pairs (Fig. 1d; Table 1). Breakpoint positions, as well as microhomology and small imbalances around the breakpoints were identical in both CCR carriers (Fig. 1d; Table 1). The two $\operatorname{der}(10)$ breakpoint junctions were also successfully amplified and sequenced in the affected son who inherited only der(10). As expected, no PCR product was observed after amplifying $\operatorname{der}(6)$ and $\operatorname{der}(7)$ translocation breakpoint junctions in the affected son (not shown).

Each CCR breakpoint disrupted known genes; single-minded family bHLH transcription factor 1 (SIM1) (NM_005068.2) (intron 2) on chr6 (1st break), glutamate ionotropic receptor kainate type subunit 2 (GRIK2) (intron 9) (NM_021956.4) on chr6 (2nd break), contactin associated protein-like 2 (CNTNAP2 or CASPR2) (intron 18) (NM_014141.5) on chr7, and protein tyrosine phosphatase, receptor type E (PTPRE) (intron 1) (NM_006504.5) on chr10. Finally, none of the identified translocation breakpoints occurred within or near any conserved non-coding cis-regulatory element regions for long-range position effects.

\section{Discussion}

Familial CCRs tend to have fewer breakpoints and are mainly maternally transmitted via oogenesis, as in the case reported by Binsbergen et al. [21] where a three-way CCR was unstably transmitted from a non-affected mother to her affected son, while de novo CCRs tend to have more breakpoints and the majority of them are paternal in origin arising during spermatogenesis [2]. Nevertheless, a few cases of familial CCRs with paternal transmission have been documented in the past leading to unbalanced or recombinant rearrangements in the offspring $[13,22]$. The fact that complex rearrangements affect spermatogenesis $[13,23]$ and, subsequently, infertility and subfertility often associated with male CCR carriers $[1,2]$, are plausible etiologies underlying this limited paternal transmission of CCRs.

In the current study, we present a rare case of familial CCR stably transmitted from a non-affected father to his non-affected daughter. Previous reports suggested that the specific CCR involved a single breakpoint on each $q$-arm of the participating chromosomes 6, 7, and 10, and the reciprocal exchange of the terminal segments created [17]. However, WG-MPS utilized in the present study allowed accurate reconstruction of the derivative chromosomes, and interestingly, revealed a cryptic translocation breakpoint on chr6 (Fig. 1b). The interstitial chr6

Table 1 Complex rearrangement breakpoint junctions as delineated by whole-genome mate-pair sequencing (WG-MPS) and Sanger sequencing (SS)

\begin{tabular}{lll}
\hline Chromosomal break & Translocation junctions as predicted by WG-MPS (GRCh37/hg19) & $\begin{array}{l}\text { Translocation breakpoint positions as } \\
\text { defined by SS (GRCh37/hg19) }\end{array}$ \\
\hline chr6 (1st break) & chr6:100899302-100900111 [TRANSLOC_BAL_18reads_chr10:129761169-129761668] & chr6:100899825-100899830 \\
chr6 (2nd break) & chr6:102274568-102275034 [TRANSLOC_BAL_13reads_chr7:147888949-147890271] & chr6:102274901-102274908 \\
chr7 & chr7:147888949-147890271 [TRANSLOC_BAL_13reads_chr6:102274568-102275034] & chr7:147889469-147889474 \\
chr10 & chr10:129761169-129761668 [TRANSLOC_BAL_18reads_chr6:100899302-100900111] & chr10:129761568-129761576 \\
\hline
\end{tabular}


segment translocated onto der(10) (Fig. 1b) was confirmed by FISH (Fig. 1c), validating the power of WG-MPS in delineating rearrangement complexity. Because of the relatively short translocation breakpoint junctions suggested by WG-MPS ( $\leq 1 \mathrm{~kb})$, breakpoint mapping to the base-pair level was feasible with the use of a single primer pair spanning each breakpoint junction. Breakpoint locations and molecular "signatures" were identical in all non-affected members, thus confirming that the CCR was stably transmitted from the father to his daughter, while malsegregation of the derivative chromosomes probably led to the inheritance of the unbalanced rearrangement in the son.

Even though a single known protein-coding gene was disrupted by each of the four CCR breakpoints in our study, such heterozygous disruption was phenotypically inconsequential. SIM1 haploinsufficiency has been associated with obesity in previous mice studies [24] and reports of patients carrying SIM1 loss-of-function variants [25-27] or chromosomal abnormalities in the SIM1 gene region [28, 29]. However, the pathogenic impact of SIM1 disruption is inconsistent as SIM1 variants have also been reported, similar to the cases presented here, in lean, control individuals $[25-27,30]$. Such phenotypic discordances can be partly explained by the presence of more complex rearrangements affecting, sometimes in addition to SIM1, other genes associated with obesity and neurodevelopmental phenotypes in affected individuals $[30,31]$ or identification of rearrangements that may protect against obesity in non-obese individuals [30]. It has also been suggested that complex gene-gene or gene-environment interactions may additionally influence the degree of the obesity phenotype penetrance [27]. Homozygous loss-of-function GRIK2 variants have been reported in patients with moderate to severe non-syndromic autosomal recessive mental retardation [32]. In addition, two de novo, heterozygous microdeletions in cis position on chromosome 6q16.1q16.2 and 6q16.3 disrupting, among others, the PRDM13 and GRIK2 genes have been reported in a patient with intellectual disability and autism; however, the authors concluded that functional interaction between both disrupted genes most probably underlies phenotype presentation [33]. Heterozygous CNTNAP2 disruptions reported in affected individuals presenting with autism spectrum disorder $[34,35]$ or Gilles de la Tourette syndrome and Obsessive Compulsive Disorder [36] were mostly located at the proximal part of the CNTNAP2 gene [34, 36] and/or were unbalanced [36], or the rearrangements were even more complex affecting other disease-candidate genes as well $[34,35]$. Homozygous CNTNAP2 variants have also been reported in affected patients with cortical dysplasia-focal epilepsy syndrome [37] or CASPR2 deficiency syndrome characterized by intellectual disability, autistic features and language impairment [38]. These examples are in contrast to those reported in healthy individuals where the CNTNAP2 gene is disrupted at more distal sites: within intron 11 by a $t(7 ; 15)$ translocation as reported by Belloso et al. [39], and within intron 18 by the CCR reported here. Thus, results from the present study support the suggestion that smaller and more distal CNTNAP2 disruptions may be phenotypically inconsequential [35]. Furthermore, we cannot exclude the possibility that the proximal and distal CNTNAP2 gene fragments are expressed as functional fusion genes with the distal and proximal GRIK2 gene fragments on $\operatorname{der}(7)$ and $\operatorname{der}(10)$, respectively, as both genes are expressed on the plus strand. Taken together, the common genetic phenomena of incomplete penetrance and variable phenotypic expression of SIM1 and CNTNAP2 disruptions, the recessive mode of inheritance of GRIK2-related phenotypes, the possibility of functional fusion gene generation, as well as the absence of additional chromosomal rearrangements affecting clinically relevant genes within the same pathways as the genes disrupted here by the CCR may explain the absence of specific clinical phenotypes in the father and daughter reported in the present study.

With the use of WG-MPS and the identification of a cryptic breakpoint, the CCR in this study was refined from a type I CCR (number of breaks = number of chromosomes) to a type IV CCR (number of breaks>number of chromosomes and there is a "middle segment"), based on the classification system proposed by Madan [3]. Specifically, the CCR here involves three chromosomes and four breakpoints, while the "middle segment" is the interstitial chr6 fragment translocated onto der(10). Type I and type IV CCRs align in different hexavalent configurations during the pachytene stage of meiosis I (Fig. 2). The cryptic chr6 breakpoint combined with possible recombination at the "middle segment" can produce new rearrangements and result in higher reproductive risk, increased unbalanced gamete production, and consequently, affected offspring [3]. More specifically, it has been estimated that there is an additional 3.5\% risk per breakpoint, whereas there is a $\sim 35 \%$ possibility for a recombination event to occur in type IV CCRs, and recombination may generally result in both unbalanced and balanced gametes [3, 21, 22].

Recent technological advances in next generation sequencing focus the investigation of chromosomal rearrangements, including CCRs, towards higher-resolution 


\section{a Type I CCR}

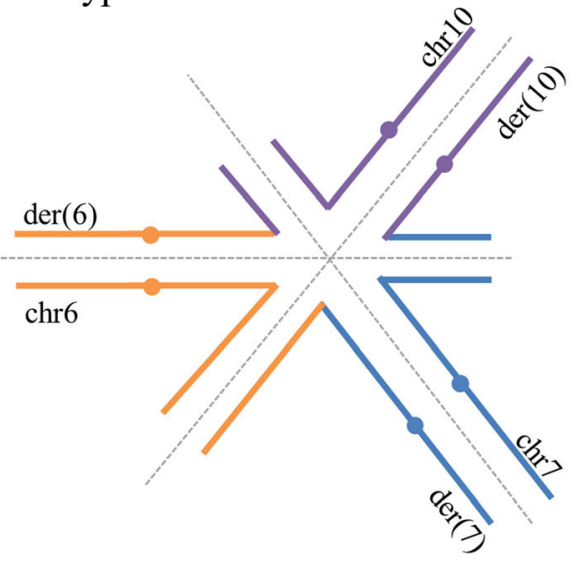

b Type IV CCR

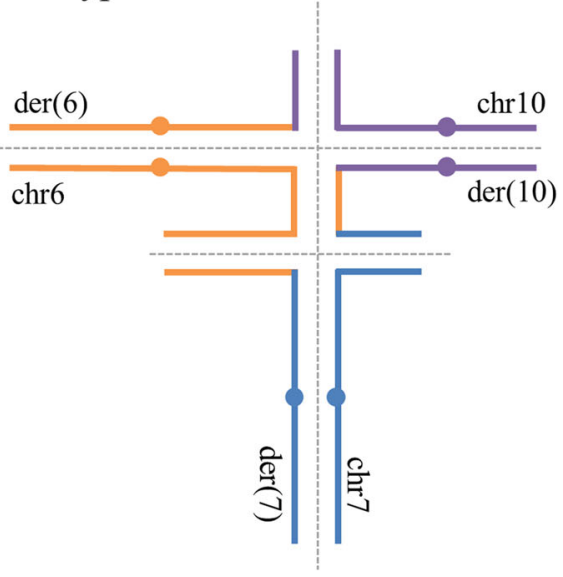

Fig. 2 Type I and Type IV CCR Hexavalent Configurations. Different hexavalent configurations in case of: a type I CCR, as determined by previous analyses, and $\mathbf{b}$ type IV CCR, as refined by whole-genome mate-pair sequencing in the current study. The additional breakpoint as well as possible recombination at the "middle segment" in type IV CCR increases the percentage of unbalanced gametes, and subsequently, reproductive risk. Genetic material from chromosomes 6, 7, and 10 are illustrated in orange, blue, and purple lines, respectively

breakpoint mapping and precise interpretation at the gene level [4]. While such approaches are highly successful in characterizing chromosomal rearrangements in detail and may reveal additional levels of complexity [15], such as in the present family reported here, conventional karyotype and molecular cytogenetic analyses remain nonetheless pivotal systemic strategies to investigate three-dimensional genome topology changes $[5,6]$. Thus, multi-level analysis using a combination of NGS and conventional cytogenetic techniques should be used instead as a holistic approach for the investigation of CCRs to gain a more complete understanding of the overall genomic system. In general, this would aid in monitoring genome instability, which can often be further induced by the use of assisted reproductive technologies, in infertile couples carrying chromosomal rearrangements [40].

\section{Conclusions}

In conclusion, the present study investigates a rare case where a phenotypically inconsequential CCR is stably transmitted from a fertile male carrier to his daughter. To the best of our knowledge, this is the first report of an apparently balanced CCR involving chromosomes 6, 7 and 10, and additional complexity discovered through WG-MPS in a family with reproductive problems. Together with previous findings, our study highlights the strength of WG-MPS as a methodology for accurate detection and characterization of CCRs. Even though the exact percentage of unbalanced gametes and reproductive risk cannot be fully determined in couples carrying CCRs, detailed characterization of individual CCRs using a combination of conventional cytogenetic and NGS-based methods remains nonetheless highly important to reveal their full complexity, as well as provide better prenatal preimplantation genetic diagnosis and genetic counseling.

\section{Abbreviations \\ ABT: Apparently balanced translocation; CCR: Complex chromosomal rearrangement; chr: Chromosome; der: Derivative; FISH: Fluorescence In-Situ Hybridization; NGS: Next generation sequencing; PCR: Polymerase Chain Reaction; WG-MPS: Whole-genome mate-pair sequencing}

\section{Acknowledgements}

We are deeply grateful to the family for participating in this study.

\section{Funding}

This work was supported by The Lundbeck Foundation [2013-14290]; the University of Copenhagen's Programme for Interdisciplinary Research (Global Genes, Local Concerns); The Danish Council for Independent Research Medical Sciences [4183-00482B]; Telethon Cyprus; and Norway Grants through the Directorate General for European Programmes, Coordination and Development of the Republic of Cyprus.

\section{Availability of data and materials}

All data generated or analysed during this study are included in this published article, or available from the corresponding author on reasonable request.

\section{Authors' contributions}

CA was responsible for the WG-MPS library preparation, interpretation of the WG-MPS results, reconstruction of the derivative chromosomes, and performed $P C R$, and Sanger sequencing. She also drafted and made final editing of the manuscript. CS was responsible for the design and coordination of the study, participated in study supervision, helped in reviewing and editing the manucript, and was responsible for experimental resources and funding acquisition. NT also participated in study supervision, and was responsible for experimental resources and funding acquisition. MB and AT contributed in the analysis of WG-MPS data and Structural Variant analysis data. MM was responsible for providing WG-MPS training to CA. AK performed and analyzed FISH data. All authors read and approved the final manuscript.

\section{Ethics approval and consent to participate}

The study was approved by the National bioethics committee as part of the Translation Facility Application (EЕВK/EП/2-13/09). Informed consents were signed from all participants. 


\section{Competing interests}

The authors declare that they have no competing interests.

\section{Publisher's Note}

Springer Nature remains neutral with regard to jurisdictional claims in published maps and institutional affiliations.

\section{Author details}

'Department of Cytogenetics and Genomics, The Cyprus Institute of Neurology and Genetics, Nicosia, Cyprus. ${ }^{2}$ The Cyprus School of Molecular Medicine, The Cyprus Institute of Neurology and Genetics, Nicosia, Cyprus. ${ }^{3}$ Wilhelm Johannsen Centre for Functional Genome Research, Department of Cellular and Molecular Medicine, University of Copenhagen, Copenhagen, Denmark.

Received: 22 January 2018 Accepted: 15 May 2018

Published online: 07 June 2018

\section{References}

1. Poot M, Haaf T. Mechanisms of origin, phenotypic effects and diagnostic implications of complex chromosome rearrangements. Mol Syndromol. 2015;6:110-34

2. Pellestor F, Anahory T, Lefort G, Puechberty J, Liehr T, Hedon B, et al. Complex chromosomal rearrangements: origin and meiotic behavior. Hum Reprod Update. 2011;17:476-94.

3. Madan K. Balanced complex chromosome rearrangements: reproductive aspects. A review. Am J Med Genet Part A. 2012;158A:947-63.

4. Schluth-Bolard C, Labalme A, Cordier M-PP, Till M, Nadeau G, Tevissen H, et al. Breakpoint mapping by next generation sequencing reveals causative gene disruption in patients carrying apparently balanced chromosome rearrangements with intellectual deficiency and/or congenital malformations. J Med Genet. 2013;50:144-50.

5. Rivera H, Ayala-Madrigal ML, Barros-Núñez JP, Arnaud-López L, Maloney V, Crolla JA. A highly complex rea(2;3;11) and aniridia by position effect. Cytogenet Genome Res. 2006:114:83-8.

6. Moralli D, Nudel R, Chan MTM, Green CM, Volpi EV, Benítez-Burraco A, et al. Language impairment in a case of a complex chromosomal rearrangement with a breakpoint downstream of FOXP2. Mol Cytogenet. 2015;8:36.

7. De Gregori M, Ciccone R, Magini P, Pramparo T, Gimelli S, Messa J, et al. Cryptic deletions are a common finding in "balanced" reciprocal and complex chromosome rearrangements: a study of 59 patients. J Med Genet. 2007:44:750-62

8. Sismani C, Kitsiou-Tzeli S, loannides M, Christodoulou C, Anastasiadou V, Stylianidou G, et al. Cryptic genomic imbalances in patients with de novo or familial apparently balanced translocations and abnormal phenotype. Mol Cytogenet. 2008;1:15.

9. López-Expósito I, Ballesta-Martinez MJ, Bafalliu JA, Vera-Carbonell A, Domingo-Jiménez R, López-González V, et al. Array CGH detection of a novel cryptic deletion at $3 q 13$ in a complex chromosome rearrangement. Genomics. 2014;103:288-91.

10. Auger J, Bonnet C, Valduga M, Philippe C, Bertolo-Houriez E, Beri-Dexheimer $M$, et al. De novo complex $X$ chromosome rearrangement unmasking maternally inherited CSF2RA deletion in a girl with pulmonary alveolar proteinosis. Am J Med Genet A. 2013;161A:2594-9.

11. Nguyen MH, Morel F, Pennamen P, Parent P, Douet-Guilbert N, Le Bris MJ, et al. Balanced complex chromosome rearrangement in male infertility: case report and literature review. Andrologia. 2015;47:178-85.

12. Lazarczyk E, Drozniewska M, Pasinska M, Stasiewicz-Jarocka B, Midro AT, Haus O. Complex balanced chromosomal translocation t(2;5;13) (p21;p15; q22) in a woman with four reproductive failures. Mol Cytogenet. 2014;7:83.

13. Gorski JL, Kistenmacher ML, Punnett HH, Zackai EH, Emanuel BS, Optiz JM, et al. Reproductive risks for carriers of complex chromosome rearrangements: analysis of 25 families. Am J Med Genet. 1988;29:247-61.

14. Aristidou C, Koufaris C, Theodosiou A, Bak M, Mehrjouy MM, Behjati F, et al. Accurate breakpoint mapping in apparently balanced translocation families with discordant phenotypes using whole genome mate-pair sequencing. PLoS One. 2017;12:e0169935

15. Kloosterman WP, Guryev V, van Roosmalen M, Duran KJ, de Bruijn E, Bakker SC, et al. Chromothripsis as a mechanism driving complex de novo structural rearrangements in the germline. Hum Mol Genet. 2011; 20:1916-24.
16. Nazaryan L, Stefanou EG, Hansen C, Kosyakova N, Bak M, Sharkey FH, et al The strength of combined cytogenetic and mate-pair sequencing techniques illustrated by a germline chromothripsis rearrangement involving FOXP2. Eur J Hum Genet. 2014;22:338-43.

17. Patsalis PC, Evangelidou P, Charalambous S, Sismani C. Fluorescence in situ hybridization characterization of apparently balanced translocation reveals cryptic complex chromosomal rearrangements with unexpected level of complexity. Eur J Hum Genet. 2004;12:647-53.

18. Li H, Durbin R. Fast and accurate short read alignment with burrowswheeler transform. Bioinformatics. 2009;25:1754-60.

19. Zeitouni B, Boeva V, Janoueix-Lerosey I, Loeillet S, Legoix-ne P, Nicolas A, et al. SVDetect: a tool to identify genomic structural variations from pairedend and mate-pair sequencing data. Bioinformatics. 2010;26:1895-6.

20. Thorvaldsdottir H, Robinson JT, Mesirov JP. Integrative Genomics Viewer (IGV): high-performance genomics data visualization and exploration. $\mathrm{Br}$ Bioinform. 2013;14:178-92.

21. van Binsbergen $E_{1}$ Hochstenbach R, Giltay J, Swinkels M. Unstable transmission of a familial complex chromosome rearrangement. Am J Med Genet Part A. 2012;158A:2888-93. Wiley-Blackwell

22. Soler A, Sánchez A, Carrió A, Badenas C, Milà M, Margarit E, et al. Recombination in a male carrier of two reciprocal translocations involving chromosomes 14, 14', 15, and 21 leading to balanced and unbalanced rearrangements in offspring. Am J Med Genet Part A. 2005;134A:309-14.

23. Kim JW, Chang EM, Song S-H, Park SH, Yoon TK, Shim SH. Complex chromosomal rearrangements in infertile males: complexity of rearrangement affects spermatogenesis. Fertil Steril. 2011;95:349-52.

24. Michaud JL, Boucher F, Melnyk A, Gauthier F, Goshu E, Levy E, et al. Sim1 haploinsufficiency causes hyperphagia, obesity and reduction of the paraventricular nucleus of the hypothalamus. Hum Mol Genet. 2001;10: 1465-73.

25. Bonnefond A, Raimondo A, Stutzmann F, Ghoussaini M, Ramachandrappa S, Bersten DC, et al. Loss-of-function mutations in SIM1 contribute to obesity and Prader-Willi-like features. J Clin Invest. 2013;123:3037-41.

26. Ramachandrappa S, Raimondo A, Cali AMG, Keogh JM, Henning E, Saeed S, et al. Rare variants in single-minded 1 (SIM1) are associated with severe obesity. J Clin Invest. 2013;123:1-9.

27. Zegers D, Beckers S, Hendrickx R, Van Camp JK, de Craemer V, Verrijken A, et al. Mutation screen of the SIM1 gene in pediatric patients with earlyonset obesity. Int J Obes. 2014;38:1000-4.

28. Holder $J \mathrm{~J}$ r, Butte NF, Zinn AR. Profound obesity associated with a balanced translocation that disrupts the SIM1 gene. Hum Mol Genet. 2000;9:101-8.

29. Faivre L, Cormier-Daire V, Lapierre JM, Colleaux L, Jacquemont S, Genevieve $D$, et al. Deletion of the SIM1 gene (6q16.2) in a patient with a Prader-Willilike phenotype. J Med Genet. 2002;39:594-6.

30. El Khattabi L, Guimiot F, Pipiras E, Andrieux J, Baumann C, Bouquillon S, et al. Incomplete penetrance and phenotypic variability of $6 q 16$ deletions including SIM1. Eur J Hum Genet. 2015;23:1010-8.

31. Kasher PR, Schertz KE, Thomas M, Jackson A, Annunziata S, Ballesta-Martinez MJ, et al. Small 6q16.1 deletions encompassing POU3F2 cause susceptibility to obesity and variable developmental delay with intellectual disability. Am J Hum Genet. 2016;98:363-72. Elsevier

32. Motazacker MM, Rost BR, Hucho T, Garshasbi M, Kahrizi K, Ullmann R, et al. A defect in the ionotropic glutamate receptor 6 gene (GRIK2) is associated with autosomal recessive mental retardation. Am J Hum Genet. 2007:81:792-8.

33. Strunk D, Weber P, Röthlisberger B, Filges I. Autism and intellectual disability in a patient with two microdeletions in 6q16: a contiguous gene deletion syndrome? Mol Cytogenet. 2016;9:88. BioMed Central

34. Bakkaloglu B, O'Roak BJ, Louvi A, Gupta AR, Abelson JF, Morgan TM, et al. Molecular cytogenetic analysis and resequencing of contactin associated protein-like 2 in autism spectrum disorders. Am J Hum Genet. 2008:82:165-73.

35. Poot M, Beyer V, Schwaab I, Damatova N, Van't Slot R, Prothero J, et al. Disruption of CNTNAP2 and additional structural genome changes in a boy with speech delay and autism spectrum disorder. Neurogenetics. 2010;11:81-9.

36. Verkerk AJ, Mathews CA, Joosse M, Eussen BH, Heutink P, Oostra BA, et al. CNTNAP2 is disrupted in a family with Gilles de la Tourette syndrome and obsessive compulsive disorder. Genomics. 2003:82:1-9.

37. Strauss KA, Puffenberger EG, Huentelman MJ, Gottlieb S, Dobrin SE, Parod JM, et al. Recessive symptomatic focal epilepsy and mutant contactinassociated protein-like 2. N Engl J Med. 2006;354:1370-7. 
38. Rodenas-Cuadrado P, Pietrafusa N, Francavilla T, La Neve A, Striano P, Vernes SC. Characterisation of CASPR2 deficiency disorder - a syndrome involving autism, epilepsy and language impairment. BMC Med Genet. 2016;17:8.

39. Belloso JM, Bache I, Guitart M, Caballin MR, Halgren C, Kirchhoff M, et al. Disruption of the CNTNAP2 gene in a $t(7 ; 15)$ translocation family without symptoms of Gilles de la Tourette syndrome. Eur J Hum Genet. 2007;15:711-3.

40. Horne SD, Abdallah BY, Stevens JB, Liu G, Ye KJ, Bremer SW, et al. Genome constraint through sexual reproduction: application of 4D-genomics in reproductive biology. Syst Biol Reprod Med. 2013;59:124-30.

Ready to submit your research? Choose BMC and benefit from:

- fast, convenient online submission

- thorough peer review by experienced researchers in your field

- rapid publication on acceptance

- support for research data, including large and complex data types

- gold Open Access which fosters wider collaboration and increased citations

- maximum visibility for your research: over $100 \mathrm{M}$ website views per year 